\title{
Estrutura de comunidades necrófagas: efeito da partilha de recursos na diversidade
}

\author{
Mauricio O. Moura ', Claudio J. B. de Carvalho ${ }^{2} \&$ Emygdio L. A. Monteiro-Filho ${ }^{2}$ \\ ${ }^{1}$ Núcleo de Pesquisas Ambientais, Departamento de Ciências Biológicas, Universidade Estadual do Centro-Oeste. \\ Caixa Postal 3010,85010-090 Guarapuava, Paraná, Brasil. E-mail: moura@unicentro.br \\ 2 Departamento de Zoologia, Universidade Federal do Paraná. Caixa Postal 19020, 81531-980 Curitiba, Paraná, Brasil. \\ E-mail: cjbcarva@ufpr.br; elamf@ufpr.br
}

\begin{abstract}
Carrion community structure: the effect of resource partitioning on diversity. Even though an extensive framework has been develop on community structuring process, there have been few tests of mechanisms promoting coexistence in local communities. We report field experiments designed to assess the role of resource partitioning as a mechanism promoting coexistence and enhancing diversity using necrophagous communities as a model system. In order to achieve this objective we let in field to be colonize, 36 carcasses with weight ranging from 17 to $440 \mathrm{~g}$, performing three repetitions. The community was composed by twenty one species of necrophagous flies. Initial analysis suggest some level of resource partitioning but there were more overlap than could be accounted by chance and the level of specialization on carcass size were very low. Based on this findings resource partitioning could not be promoting coexistence and another mechanism should be invoked to account for such effect.
\end{abstract}

KEY WORDS. Coexistence, ephemeral communities, null model.

RESUMO. Mesmo tendo sido desenvolvido um arcabouço teórico dos processos que estruturam as comunidades, existem poucos testes dos mecanismos que promovem a coexistência em comunidades locais. Neste trabalho é descrito um experimento de campo desenhado para determinar o papel da partilha de recursos como um mecanismo promotor da coexistência e do aumento da diversidade usando comunidade necrófagas como modelo. Para realizar este estudo foram deixadas no campo para que fossem colonizadas, 36 carcaças com peso variando entre 17 e $440 \mathrm{~g}$ em um experimento com três repetições. A comunidade é composta de 21 espécies de moscas necrófagas. As análises iniciais sugeriam partilha de recursos mas existe mais sobreposição do que é esperado ao acaso e o nível de especialização em relação ao tamanho da carcaça foi baixo. Assim, as evidências indicam que a partilha de recursos não pode ser o principal mecanismo promotor da coexistência, considerando o tamanho das carcaças utilizadas.

PALAVRAS CHAVE. Coexistência, comunidades efêmeras, modelos nulos.

A competição interespecífica tem sido uma das teorias mais difundidas para explicar a estrutura local de comunidades, sendo que uma série de estudos tem demonstrado que as interações interespecíficas são o mecanismo responsável pela manutenção da diversidade em vários grupos animais (CoNNell 1983, Schoener 1982, Gotelli \& McCabe 2002). Em geral, duas formas de análise são utilizadas para determinar o papel da competição interespecífica na estruturação das comunidades em condições limitantes, experimentos de manipulação e análise dos padrões de uso dos recursos. Os experimentos são desenhados para manipular as densidades das espécies constituintes do sistema onde espera-se que, caso ocorra competição, a densidade da espécie competitivamente inferior seja alterada na presença da espécie competitivamente mais agressiva. Por outro lado, as análises de utilização de recursos baseiam-se na premissa de que deve existir uma padrão de uso que minimize as interações e por conseguinte aumente a possibilidade de coexistência. Neste contexto, existe a necessidade de que as espécies constituintes do sistema partilhem um recurso limitante, minimizando os efeitos das interações.

A relação entre sobreposição no uso de recursos limitantes com a existência e magnitude da competição ainda não é claramente estabelecido. Ao estudarem a coexistência em lagartos do gênero Anolis Daudin, 1802 nas Antilhas, Pacala \& Roughgarden (1985) demostraram que as interações competitivas ocorriam em áreas com alta sobreposição no uso dos recursos mas não em áreas onde a sobreposição era baixa. Porém, em outro estudo, HAIRSTON (1980) encontrou justamente o oposto, ou seja maiores interações competitivas em áreas com a menor sobreposição no uso de recursos. A discrepância nestes resultados pode ser devi- 
do a vários fatores como escala espacial escolhida, recursos considerados ou diferenças intrínsecas nos táxons, embora teoricamente, desde que os recursos sejam bióticos (ARMSTRONG \& McGenee 1980) ambos os resultados são possíveis, desde que mediados por interações indiretas (Abrams 1998). Já, no caso de recursos abióticos, teoricamente só existe uma possibilidade, a de que a competição aumente a medida que a sobreposição aumente (AвRAMs 1998).

Comunidades de invertebrados associados à matéria orgânica em decomposição, são normalmente constituídas por duas a três espécies consideradas dominantes (BEAver 1977, HansKi \& KuUsela 1977, Kneidel 1984, BraAck 1987, Souza \& Linhares 1997, WoDсоок et al. 2002) com uma tendência de aumento da riqueza à medida que aumentam o tamanho da carcaça ou o número de carcaças amostradas. Este padrão ocorre na grande maioria dos estudos, o que sugere um mecanismo comum para a estruturação e manutenção da diversidade nestas comunidades. Os insetos necrófagos são atraídos para a carcaça através de odores oriundos do processo de decomposição. Após encontrar a carcaça, ocorre a oviposição/larviposição, com as larvas desenvolvendo-se neste ambiente que é a arena onde as interações ocorrerão, dada sua natureza discreta no tempo e no espaço (Finn 2001).

Existe uma grande quantidade de evidências tanto de experimentos em laboratório (PrinkKILA \& HANSKi 1995, Zuben et al. 1993, Reis et al. 1994, 1996) quanto de campo (Wodcook et al. 2002) sobre a existência e o efeito de interações competitivas nestas comunidades. Por outro lado, apesar da evidência de interações, estas comunidades têm diversidade e riqueza elevadas (Beaver 1977, Hanski \& Kuusela 1977, Moura 2004). Estas duas características, interações competitivas potenciais e alta riqueza, induzem à busca dos mecanismos que mantêm a diversidade nestas comunidades. Duas hipóteses principais têm sido consideradas: partilha de recursos e agregação de competidores.

Ao estudar a estrutura de uma guilda de moscas necrófagas, Moura (2004) concluiu que todas as espécies pertencentes ao sistema estavam distribuídas de maneira agregada e que esta agregação facilitaria a coexistência. No entanto, não considerou outros mecanismos, que também poderiam levar ao padrão espacial encontrado, como uma possível especialização de algumas espécies do sistema em recursos com tamanhos diferentes. Denno \& Cothran (1975) consideraram o tamanho e o tipo da carcaça como sendo um dos três eixos mais importantes para a partilha de recursos em comunidades necrófagas. Há uma evidência empírica fraca sobre as influências do tamanho e do tipo da carcaça na estrutura das comunidade necrófagas, principalmente porque os estudos precedentes foram conduzidos com número limitado de espécies (Denno \& Cothran 1975) ou não compararam a sobreposição com uma hipótese nula apropriada (KuUsela \& Hanski 1982, Kneidel 1984). Com relação ao tamanho da carcaça uma generalização parece constante, a que a riqueza aumenta positivamente com o tamanho da carcaça (DenNo \& Cothram 1975, Wells \& GreEnberg 1994), embora existam resultados contrários, como os de KuUsela \& HANSKI (1982). Já a exis- tência de especialização não possui um padrão claro, sendo que Denno \& Cothram (1975) e Wells \& Greenberg (1994) comprovaram experimentalmente que pelo menos algumas espécies demonstram preferência por carcaças grandes ou pequenas. Já, no experimento realizado por KuUsela \& HANSKI (1982), a variação entre as classes de tamanho das carcaças não foi diferente do que se esperaria por acaso, indicando uma colonização aleatória. No entanto, existe uma característica presente em todos os resultados que é a sobreposição entre as espécies na utilização das classes de recurso, que pode em alguns casos ser considerada alta, embora nenhum dos trabalhos citados anteriormente tenha considerado este fator de maneira estatística.

O significado do tamanho da carcaça na coexistência das espécies pode ser avaliado medindo-se o grau de especialização e de sobreposição entre as espécies e então comparando estas medidas às predições baseadas em modelos nulos oriundos da teoria da competição interespecífica. Dadas estas considerações, neste trabalho será estudado o papel da partilha de recursos na estrutura da comunidade baseando-se na possibilidade de estruturação através de colonização diferencial de carcaças de tamanhos diferentes. Especificamente, testaremos o seguinte: 1) há uma especialização em relação ao tamanho da carcaça?; 2) Qual é a quantidade de sobreposição entre as espécies?; 3) A sobreposição observada é maior ou menor do que se espera em uma colonização aleatória?

\section{MATERIAL E MÉTODOS}

Para se determinar o efeito do tamanho da carcaça na distribuição da espécies necrófagas foi delineado um experimento com três repetições. O experimento foi realizado em um local aberto situado dentro do campus Centro Politécnico da Universidade Federal do Paraná em Curitiba $\left(25^{\circ} 25^{\prime} \mathrm{S}, 49^{\circ}\right.$ $\left.25^{\prime} \mathrm{W}\right)$. Neste ambiente, a vegetação consiste de um mosaico de campo, composto principalmente por arbustos e árvores dispersas, e áreas de brejo.

O experimento consistiu em fornecer para colonização 36 carcaças de ratos (Rattus norvergicus Berkenhout, 1769) com pesos variando entre 17 e $440 \mathrm{~g}$. Foram estabelecidas cinco categorias de peso: Classe A (< $50 \mathrm{~g})$; Classe B (50-99 g); Classe C (100-149); Classe D (150-199 g) e Classe E ( $\geq 200$ g). Após serem sacrificados por deslocamento cervical, os animais foram aleatoriamente alocados em uma célula de uma grade medindo 50 x $60 \mathrm{~m}$ da seguinte maneira: dez carcaças pequenas ( $<100 \mathrm{~g}$ ) foram alocadas isoladamente em copos plásticos dentro de uma armadilha para coleta de adultos; dois grupos de dez carcaças pequenas foram alocadas dentro de gaiolas de arame (usadas para exclusão de necrófagos de porte médio e grande) além de quatro carcaças médias (100-200 g) e duas grandes (> $200 \mathrm{~g}$ ) colocadas isoladamente em gaiolas de arame. O desenho acima permitia avaliar também o efeito do agrupamento do recurso (carcaças agrupadas x não agrupadas) no padrão de ocupação da comunidade. Os copos plásticos tiveram 1/3 do seu volume preenchido por vermiculita e as gaiolas possuíam 
uma bandeja localizada abaixo da carcaça também preenchida parcialmente com vermiculita.

Após a instalação do experimento, as armadilhas foram vistoriadas diariamente, sendo os adultos coletados em cada visita. As carcaças colocadas nos copos plásticos foram trazidas para o laboratório assim que tivessem perdido aproximadamente $1 / 3$ do seu peso. Em cada gaiola, as bandejas eram vistoriadas diariamente para procura de larvas que eram então, coletadas. Todas as larvas foram mantidas em uma casa de vegetação sob condições equivalentes às de campo até a emergência dos adultos, que foram identificados e contados.

Para avaliar a possível existência de preferência por tamanho de carcaça no momento da oviposição, foi utilizado o índice de especialização de nicho de Levins (B), o qual é calcu-

lado da seguinte maneira: $B=1 / \sum p_{j}^{2}$ onde: $\mathrm{p}_{\mathrm{j}}$ é a proporção de indivíduos que utilizam o recurso do tipo $j$, o qual corresponde a uma das classes de tamanho. Após o cálculo, todas as medidas foram estandardizadas usando a seguinte expressão (Krebs 1989): $B_{a}=B-1 / n-1$ onde: $B$ refere-se ao valor do índice de Levins e $n$ ao tamanho amostral.

A análise da sobreposição de nicho foi feita considerando o tamanho da carcaça (dividido em classes como descrito previamente) como variável a ser partilhada. Assim, o valor da sobreposição reflete a porcentagem de uso das diferentes classes de recursos entre as espécies do sistema, tomadas par a par e inferidas a partir da abundância registrada em cada classe de tamanho. Para a análise da sobreposição do nicho, ou seja da porcentagem de utilização de uma determinada classe de recurso por duas espécies, foi utilizado o índice de Czechanowski que representa a fração de indivíduos de duas espécies que emergem de um mesmo recurso e é calculado desta forma: $O_{12}=O_{21}=1-0,5 \sum_{i=1}^{K}\left|p_{1 i}-p_{21}\right|$ onde: $O$ é a medida de sobreposição entre as espécies 1 e 2e $p$ é a proporção de indivíduos emergindo em $i(i . . . k)$ classes de recurso, que correspondem às classes de peso utilizadas.

Em uma comunidade estruturada por competição interespecífica espera-se que as espécies possuam uma sobreposição mais baixa do que em um cenário em que a competição não ocorre. Para se testar esta premissa sobre o que se esperaria de uma comunidade estruturada por competição interespecífica, foi construído um modelo nulo para que fosse possível comparar os valores encontrados no campo com valores gerados aleatoriamente. Para a análise do modelo nulo foi utilizado o Programa Ecosim 7.0 (Gotelli \& ENTSMinger 2001). O algoritmo utilizado retém o valor do nicho calculado para cada espécie mas aleatoriza como as classes de recurso são utilizadas. Desta forma, nas matrizes geradas na simulação, a utilização global dos recursos pelas espécies é a mesma que na matriz original (emergem do recurso na mesma proporção), mas os valores agora são independentes. Após a matriz simulada ter sido gerada, a sobreposição era calculada conforme descrito acima. Após 1.000 simulações, a média e a variância dos valores simulados foram comparadas com os valores observados e computados os valores das probabilidades associadas.

O efeito da réplica, da posição da armadilha na grade e do tamanho da carcaça na abundância e densidade (número de indivíduos por grama de peso da carcaça) das espécies foi avaliada usando análise de variância (Anova e Kruskall-Wallis - KW) e regressão linear (ZAR 1984). Estes testes foram executados usando o software Statistica (STATSOFT 1996).

\section{RESULTADOS}

Durante o experimento foram coletados 1082 adultos pertencentes a 30 espécies. Destes, somente três espécies, Lucilia eximia (Wiedemann, 1819), Chrysomya albiceps (Wiedemann, 1819) e Oxysarcodexia paulistanensis (Mattos, 1919) representam quase $80 \%$ de todos os indivíduos coletados. As espécies não tiveram sua captura influenciada pela posição da carcaça na grade $(\mathrm{KW}=11,87 ; \mathrm{p}=0,22)$ e ocorreu uma relação positiva entre abundância e a fração de ocupação dos recursos (carcaças) ( $\mathrm{r}=0,67 ; \mathrm{p}<0,001)$. Em laboratório, emergiram 15410 indivíduos pertencentes a 21 espécies (Tab. I), das quais três foram identificadas até gênero e portanto serão consideradas ao nível de Família, Sphaeroceridade (Leptocera Olivier, 1813 e Coproica, Rondani, 1861) e Piophilidae (Piophila Fallen, 1810). Destas, duas espécies criadas não foram coletadas nas armadilhas para adultos. Existe uma diferença significativa $\left(\mathrm{F}_{2,223}=8,20\right.$; $\mathrm{p}<0,001$ ) entre as réplicas com relação à riqueza e abundância das espécies que foram criadas, sendo que a primeira fase de campo é a que possui a maior riqueza e abundância (Tab. I).

O tamanho da carcaça teve uma influência significativa na variação da abundância entre as espécies $\left(\mathrm{KW}_{4,84}=44.71 ; \mathrm{p}\right.$ $<0,001)$. Graficamente, é possível dividir as classes do tamanho em dois grupos (Fig. 1) com a classe de 100 g sendo o limite entre os grupos. Da mesma forma, existe um efeito significativo do tamanho da carcaça na riqueza $\left(\mathrm{KW}_{4,84}=17,11 ; \mathrm{p}\right.$ $=0,001$ ) de tal forma que carcaças com mais de $100 \mathrm{~g}$ possuem, em média, mais espécies do que carcaças abaixo deste limite (Fig. 1). O número total de espécimes que emergiram possui uma relação positiva, embora fraca, com o tamanho da carcaça $(\mathrm{r}=0,44 ; \mathrm{p}=0,04 ;$ Fig. 2). Já, a densidade possui um padrão diferente, sendo negativamente relacionada com o tamanho da carcaça ( $\mathrm{r}=-0,14 ; \mathrm{p}=0,19 ;$ Fig. 3$)$.

O grau de especialização entre as espécies necrófagas variou de zero a 0,87 , com um valor médio de $0,21 \pm 0,25$. Os valores do índice estão bastante dispersos ao longo da escala total, como indica sua variância alta (Fig. 4). Um valor zero significa que as moscas utilizam para reprodução somente um tamanho da carcaça, valores entre 0,30 e 0,50 agrupam espécies que se reproduziram em duas classes e valores maiores do que 0,50 espécies que se reproduziram em mais de três classes de tamanho. Onze espécies se reproduziram em duas ou mais classes do recurso. As espécies que se reproduziram em uma única classe do recurso (especialistas) utilizaram principalmente das 


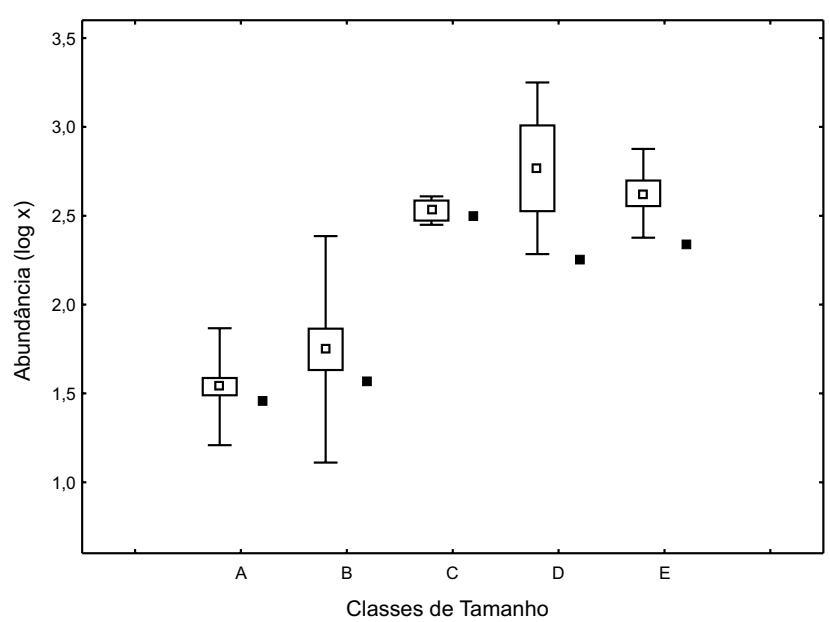

Figura 1. Abundância (quadrados claros) e riqueza (quadrados escuros) das espécies criadas em carcaças de ratos em relação as classes de Peso. As Classe de peso são Classe A (<50 g); Classe B (50-99 g); Classe C (100-149 g); Classe D (150-199 g) e Classe E (> 200 g). Médias (quadrados), erro padrão e desvio padrão (barras).

carcaças pequenas (classes A e B). As espécies que foram criadas em mais de três classes do peso não mostraram nenhuma tendência.

A análise da sobreposição de nicho reflete a fração dos indivíduos que emergem do mesmo recurso. O resultado indica que a maioria das comparações entre pares de espécies tem valores acima de 50\% de sobreposição. Apesar destes valores de sobreposição serem altos, a sobreposição média global foi de $37 \%$. A comparação entre os valores observados e simulados da sobreposição do nicho gerados pelo modelo nulo mostrou que os valores observados eram significativamente diferentes do esperado por acaso e posicionado acima da distribuição gerada (Fig. 5, Tab. II). Esta diferença indica que o padrão da sobreposição nesta guilda tem uma probabilidade muito pequena de ocorrer por acaso. Da mesma maneira, o valor da variância observada era muito superior ao valor da variância simulada (Tab. II).

\section{DISCUSSÃO}

A competição interespecífica é um fenômeno que possui um bom registro de observações e da experimentações nas comunidades necrófagas, onde as espécies são envolvidas em competição por exploração (NICHOLSON 1957). Estas interações produzem geralmente efeitos deletérios nos componentes da aptidão (ReIs et al.1994, PRINKKILA \& HANSKI 1995) e portanto influências na dinâmica da população (ZuBEn et al. 1993, ReIs et al. 1996). Desde que o recurso, carcaça, é sempre um fator limitante para a comunidade necrófaga (HANSKI 1987a), é possível usar as premissa da teoria do nicho com uma estrutura analítica. De acordo com a teoria da partilha de recursos, as espécies atuais no sistema devem expressar algum tipo de diferença devido a especiali-
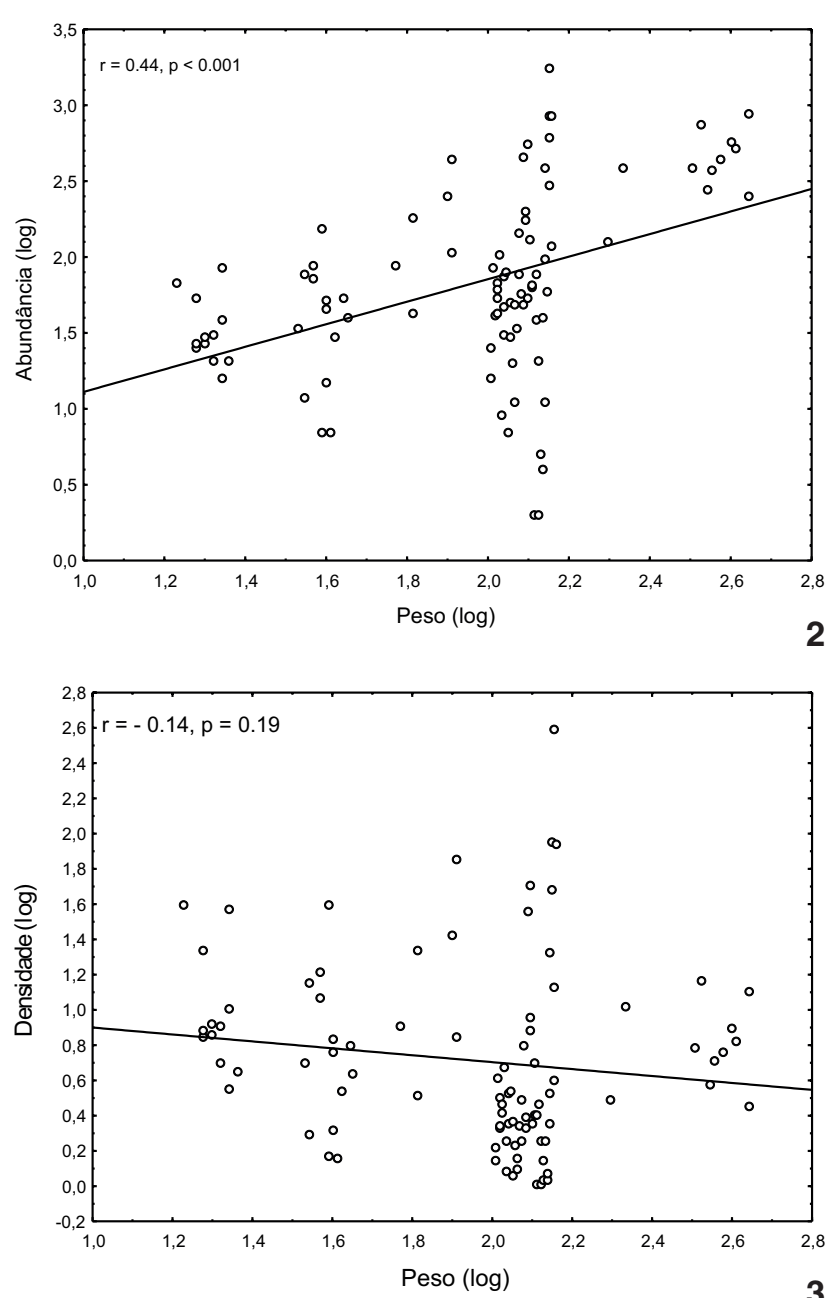

Figuras 2-3. Relação entre o tamanho da carcaça (peso) e abundância das espécies necrófagas (2) e relação entre tamanho da carcaça (peso) e a densidade (número de indivíduos criados por grama de peso) das espécies necrófagas (3). Ambos os eixos estão em escala logarítmica.

zação em determinados tipos/classes de recurso, produzindo uma medida de sobreposição significativamente inferior do que o esperado por acaso (SCHOener 1974, 1990, Roughgarden 1983, PIANKA 1996). Os resultados descritos neste trabalho não são consistentes com estas predições. A medida da amplitude do nicho indica um grau baixo de especialização, o que não seria esperado se as fêmeas fossem seletivas de acordo com o tamanho da carcaça. As espécies generalistas, que se reproduziram em três ou mais classes do tamanho, incluem as espécies mais abundantes na área e as especialistas, que se reproduziram em somente em uma classe do tamanho, ocorreram somente em carcaças pequenas. Mesmo estas espécies (dez no total) não podem ser consideradas especialistas verdadeiras, já que pelo menos seis destas já 

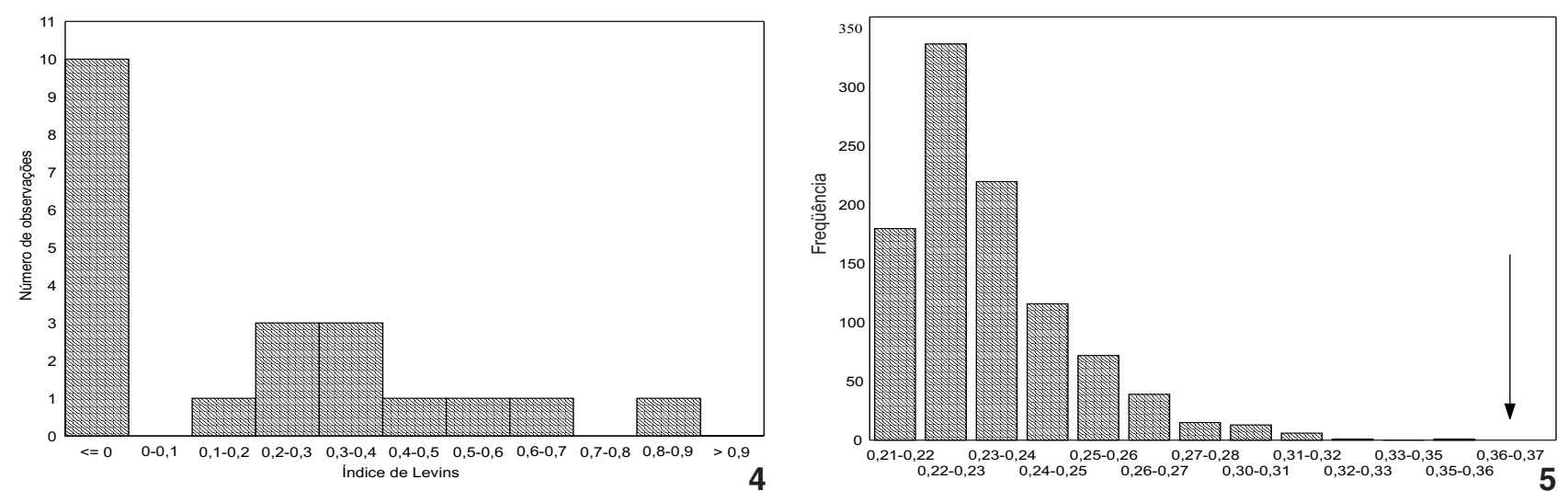

Figuras 4-5. (4) Distribuição de freqüência dos valores do índice de Levins calculadas entre pares de espécies; (5) histograma de freqüência dos valores simulados (1000 aleatorizações) do índice de sobreposição de Czechanowski indicando (flecha) a posição do valor observado de sobreposição.

Tabela I. Composição de espécies e freqüência de ocorrência de Diptera necrófagas que se criaram em carcaças de ratos durante janeiro (repetição I), fevereiro (repetição II) e março (repetição III) de 1999 em Curitiba, Paraná.

\begin{tabular}{|c|c|c|c|c|}
\hline Táxons & Janeiro & Fevereiro & Março & Total \\
\hline \multicolumn{5}{|l|}{ Calliphoridae } \\
\hline Chrysomya albiceps (Wiedemann, 1819) & 542 & 524 & 311 & 1377 \\
\hline Chrysomya megacephala (Fabricius, 1794) & 19 & - & - & 19 \\
\hline Lucilia eximia (Wiedemann, 1819) & 4738 & 2877 & 4647 & 12262 \\
\hline Lucilia sericata (Meigen, 1826) & 6 & - & 67 & 73 \\
\hline Paralucilia xanthogeneiates Dear, 1985 & - & - & 33 & 33 \\
\hline Sarconesia chlorogaster (Wiedemann, 1830) & 109 & - & 11 & 120 \\
\hline Hemilucilia semidiaphana (Rondani, 1850) & 43 & - & 44 & 87 \\
\hline \multicolumn{5}{|l|}{ Muscidae } \\
\hline Ophyra aenescens (Wiedemann, 1830) & 55 & - & - & 55 \\
\hline Ophyra chalcogaster (Wiedemann, 1824) & 27 & - & - & 27 \\
\hline Sarcopromusca pruna (Shannon \& del Ponte, 1926) & 50 & - & - & 50 \\
\hline \multicolumn{5}{|l|}{ Fanniidae } \\
\hline Fannia femoralis Stein, 1898 & - & 41 & - & 41 \\
\hline Fannia pusio Wiedemann, 18830 & 17 & - & - & 17 \\
\hline \multicolumn{5}{|l|}{ Anthomyiidae } \\
\hline Craspedochaeta punctipennis Wiedemann, 1830 & - & 4 & - & 4 \\
\hline \multicolumn{5}{|l|}{ Sarcophagidae } \\
\hline Helicobia aurescens (Towsend, 1927) & 15 & - & - & 15 \\
\hline Towsendmyia halli (Engel, 1931) & 222 & 150 & 635 & 1007 \\
\hline Sarcodexia lambens (Wiedemann, 1830) & 34 & 27 & - & 61 \\
\hline Pattonela resona (Lopes, 1935) & 61 & - & - & 61 \\
\hline Oxysarcodexia paulistanensis (Mattos, 1919) & 9 & - & 20 & 29 \\
\hline Sphaeroceridae & 49 & & & 49 \\
\hline Piophilidae & 23 & & & 23 \\
\hline
\end{tabular}

foram criadas em carcaças muito maiores (Moura et al. 1997, Souza \& Linhares 1997), inclusive na mesma área (M. O. Moura, dados não publicados). O que pode estar ocorrendo é a utiliza- ção de uma fração de recurso que estava disponível, embora para várias espécies de Diptera dados de comportamento de oviposição não existem, o que dificulta delimitar claramente um padrão. 
Tabela II. Estatísticas descritivas (observadas e esperadas) derivadas das simulações de aleatorização dos padrões de sobreposição na utilização do recurso. Os valores esperados foram baseados em 1000 aleatorizaçõ̃es dos padrões de sobreposição de nicho.

\begin{tabular}{|c|c|c|c|c|c|}
\hline \multicolumn{3}{|c|}{ Média } & \multicolumn{3}{|c|}{ Variância } \\
\hline Observada & Simulada & Probabilidade & Observada & Simulada & Probabilidade \\
\hline 0,36 & 0,24 & $p<0,001$ & 0,067 & 0,053 & $p=0,001$ \\
\hline
\end{tabular}

Os valores observados de sobreposição entre as espécies são altamente improváveis de ocorrer apenas por acaso, mas em uma direção contrária à esperada pela teoria da competição interespecífica (SCHOENER 1990). Este resultado demonstra que, mesmo que exista preferência por determinado tamanho de carcaça, esta escolha não produziu uma diminuição na sobreposição entre as espécies necrófagas. Assim, a variação da composição da comunidade em resposta a diferenças no tamanho na carcaça não manteria sozinha a diversidade. As variâncias observadas foram significativamente maiores que as obtidas nas aleatorizações. Este fato deve representar algum tipo de estruturação interna à comunidade ou, alternativamente, pode apenas refletir a variação espacial atual no sistema (HANSKI 1982, Moura 2004). No entanto, estes resultados estão baseados na análise de sobreposição em carcaças pequenas e médias sendo que a inclusão de carcaças maiores (aumentando o espectro) pode alterar a dinâmica geral.

Os resultados sobre a distribuição dos adultos mostraram que a colonização pareceu ser executada aleatoriamente, já que não houve preferência com relação à posição das carcaças na grade. Esta distribuição aleatória pode também ser vista como uma demonstração que não ocorre especialização relacionada ao tamanho da carcaça e que a sobreposição deve realmente ser grande. De fato, estudos precedentes (CoRnaby 1974, Beaver 1977, KuUsela \& Hanski 1982, Blackith \& Blackith 1990, Kentner \& Streit 1990) apresentaram o mesmo padrão, com uma sobreposição grande entre as espécies na utilização do recurso e colonização aleatória das carcaças, embora nestes estudos não houve uma comparação direta com uma distribuição aleatória. Além disso, mesmo em trabalhos que consideram a partilha de recursos como um importante fator para a estruturação da comunidade, assim como o relatado por KNEIDEL (1984), a sobreposição entre as espécies estudadas é alta. Esta alta taxa de sobreposição nos padrões de uso do recurso poderiam estar relacionados a correlação positiva encontrada entre abundância e ocupação das carcaças. Esta relação implica em que as espécies com abundância mais alta são as espécies com maior amplitude de distribuição na área e que portanto devem ser encontradas em mais carcaças (HANSKI 1982). Assim, estas espécies devem se sobrepor mais do que o esperado ao acaso com as outras espécies caso, não exista especialização em algum eixo do recurso.

$\mathrm{O}$ padrão de estrutura de comunidade geralmente observado em comunidades necrófagas é caracterizado pela dominância de duas ou três espécies (BRAACK 1987, HANSKI 1987b, Blackith \& Blackith 1990, Moura et al. 1997, Souza \& Linhares
1997, Woodcook et al. 2002). Nossos resultados são consistentes com este padrão, apesar da riqueza ser maior em nossas amostras. A relação entre o tamanho da carcaça e abundância/ densidade mostrou que as carcaças maiores produzem mais indivíduos mas em uma densidade mais baixa, uma combinação que pode direcionar a comunidade à dominância de poucas espécies com poder competitivo maior. Este tipo do correlação pode ser encontrada em estudos anteriores de comunidades necrófagas (Denno \& Cothran 1975, KuUsela \& Hanski 1982, KNEIDEL 1984, BRAACK 1987) bem como em outras comunidades efêmeras (Sevenster \& van Alphen 1996).

De maneira geral, os resultados apontam para ausência de especialização em relação ao tamanho da carcaça bem como para uma taxa de sobreposição entre os pares de espécies superior a que se esperaria aleatoriamente. Este cenário, baseado na distribuição de classes de peso usadas, contraria o que se esperar em caso de estruturação via partilha de recursos, indicando que este não é um fator primordial para a manutenção da diversidade em comunidades necrófagas.

\section{AGRADECIMENTOS}

A Marcio R. Pie e José Marcelo R. Aranha pelas críticas ao manuscrito e a Sandro Menezes da Silva pela ajuda com a descrição da área. A José Roberto Pujol Luz e um revisor anônimo pelas sugestões ao trabalho.

\section{REFERÊNCIAS BIBLIOGRÁFICAS}

Aвrams, P.A. 1998. High competition with low similarity and low competition with high similarity: Exploitative and apparent competition in consumer-resource systems. American Naturalist, Chicago, 152 (1): 114-128.

Armstrong, R.A. \& R. McGehee. 1980 Competitive exclusion. American Naturalist, Chicago, 115 (2): 151-170.

Beaver, R.A. 1977. Non-Equilibrium 'Island' Communities: Diptera breeding in dead snails. Journal of Animal Ecology, London, 46 (3): 783-798.

BLACKITH, R.E. \& R.M. BLACKITH. 1990. Insect infestation of small corpses. Journal of Natural History, London, 24 (3): 699 709

BRAACK, L.E.O. 1987. Community dynamics of carrion attendant arthropods in tropical african woodland. Oecologia, Berlin, 72 (4): 402-409

ConNell, J.H. 1983. On the prevalence and relative importance of interspecific competition: evidence from field experiments. 
American Naturalist, Chicago, 122 (5): 661-696.

Cornaby, B.W. 1974. Carrion Reduction by animals in contrasting tropical habitats. Biotropica, Oxford, 6: 51-63

DenNo, R.F. \& W.R. Cothran. 1975. Niche relationships of a guild of necrophagous flies. Annals of the Entomological Society of America, Lanhan, 68 (4): 741-754

FinN, J. A. 2001. Ephemeral resource patches as model systems for diversity function experiments. Oikos, Copenhagen, 92 (2): 363-366.

GotelLi, N.J. \& G.L. ENTSMinger. 2001. EcoSim: null models software for ecology. Acquired Intelligence Inc. \& Kesey-Bear, version 7.0, Available on line at: http://homepages. together.net/ gentsmin/ecosim.htm

Gotelli, N.J. \& D.J. McCabe. 2002. Species co-occurrence: a metaanalysis of J.M. Diamond's assembly rules model. Ecology, Washington, 83 (8): 2091-2096

Hairston, N.G. 1980. The experimental test of an analysis of field distributions: competition in terrestrial salamanders. Ecology, Washington, 6 (5): 817-826.

HANSKI, I. 1982. Dynamics of regional distribution: the core and satellite hypothesis. Oikos, Copenhagen, 38 (2): 210-221.

HANsKI, I. 1987a. Colonization of ephemeral habitats, p. 155185. In: A.J. Gray; M.J. Crawley \& P.J. Edwards (Eds). Colonization, Succession and Stability. London, Blackwell Scientific Publications, 482p.

HANSKI, I. 1987b. Carrion fly community dynamics: patchiness, seasonality and coexistence. Ecological Entomology, London, 12 (3): 257-266.

Hanski, I. \& S. KuUsela. 1977. An experiment on competition and diversity in the carrion fly community. Annals of Entomology Fennici, Helsinki, 43 (1): 108-115.

Kentner \& B. Streit. 1990. Temporal distribution and habitat preference of congeneric insect species found at rat carrion. Pedobiologia, Oldenburg, 34 (6): 347-359

KNeIDel, K.A. 1984. Competition and disturbance in communities of carrion-breeding Diptera. Journal of Animal Ecology, London, 53 (4): 849-865.

Krebs, C.J. 1989. Ecological Methodology. New York, Harper \& Row Publishers, XI+654p.

KuUSELA, S. \& I. HANSKI. 1982. The structure of carrion fly communities: the size and the type of carrion. Holartic Ecology, Lund, 5 (4): 337-348.

Moura, M.O. 2004. variação espacial como mecanismo promotor da coexistência em comunidades de insetos necrófagos. Revista Brasileira de Zoologia, Curitiba, 21 (3): 409-669.

Moura, M.O.; C.J.B Carvalho \& E.L.A. Monteiro-Filho.1997. A preliminary analysis of insects of medico-legal importance in Curitiba, Paraná. Memórias do Instituto Oswaldo Cruz, Rio de Janeiro, 92 (2): 269-274.

Nicholson, A.J. 1957. The self-adjustment of populations to change. Cold Spring Harbor Symposium in Quantitative Biology, Cold Spring Harbor, 22 (3): 153-173.

Pacala, S.W. \& J. Roughgarden. 1985. Population experiments with the Anolis lizards of St. Maarten and St. Eustatius. Ecology, Washington, 66 (2):129-141.

PIANKA, E. R. 1996. Evolutionary ecology. New York, Harper \& Collins, $5^{\text {th }}$ ed., $486 \mathrm{p}$.

PrinkKila M.L. \& I. Hanski. 1995. Complex competitive interactions in four species of Lucilia blowflies. Ecological Entomology, London, 20 (3): 261-272

Reis, S.F.; G. Stangenhaus; W.A.C. Godoy; C.J. von Zuben \& O.B. RiBeiro. 1994. Variação em Caracteres Bionômicos em função da Densidade Larval em Chrysomya megacephala e Chrysomya putoria (Diptera, Calliphoridae). Revista brasileira de Entomologia, São Paulo, 38 (4): 33-46.

Reis, S.F.; M.A. Teixeira; F.J.von Zuben; W.A.C. Godoy \& C.J.von Zuben. 1996. Theoretical Dynamics of Experimental Populations of Introduced and Native Blowflies (Diptera: Calliphoridae). Journal of Medical Entomology, London, 33 (1): 537-544.

Roughgarden, J. 1983. Competition and theory in community ecology. American Naturalist, Chicago, 122 (5): 583-601.

Schoener, T.W. 1974. Resource Partioning in Ecological Communities. Science, Washington, 185 (1): 27-39.

Schoener, T. W. 1982. The controversy over interspecific competition.American Naturalist, Chicago, 70 (6): 586-595.

Schoener, T. W. 1990. The ecological Niche, p. 79-113. In: J.M. Cherret (Ed). Ecological Concepts. London, Blackwell Scientific Publications, 385p.

Sevenster, J.G. \& J.J.M. van Alphen. 1996. Aggregation and coexistence. II. A neotropical Drosophila community. Journal of Animal Ecology, London, 65 (2): 308-324.

SouzA, A. M. \& A.X. Linhares. 1997. Diptera and Coleoptera of potential forensic importance in Southeastern Brazil: relative abundance and seasonality. Medical and Veterinary Entomology, London, 11 (1): 8-12.

STATSOFt. 1996. STATISTICA for windows. Tulsa, computer program manual, version 5.1.

Wells, J.D. \& B. Greenberg. 1994. Resource use by an introduced and native carrion flies. Oecologia, Berlin, 99 (2): 181-187

Woodcock, B.A.; A.D. Watt \& S.R. Leather. 2002. Aggregation, habitat quality and coexistence: a case study on carrion fly communities in slug cadavers. Journal of Animal Ecology, London, 71 (1): 131-140.

ZAR, J.H. 1984. Biostatistical Analysis. Englewood Cliffs, Prentice-Hall International, $2^{\text {nd }}$ ed., 663p.

Zuben, C.J. von; S.F. dos Reis; J.B.R. VaL; W.A.C. Godoy \& O.B. Ribeiro. 1993. Dynamics of a mathematical model of Chrysomya megacephala (Diptera: Calliphoridae). Journal of Medical Entomology, Lanhan, 30 (2): 443-448.

Recebido em 24.V.2005; aceito em 18.XI.2005.

Revista Brasileira de Zoologia 22 (4): 1134-1140, dezembro 2005 